\title{
Impact of the external e-commerce on monopoly
}

\author{
Xiao-xing Liu \\ School of Economics and Management, Southeast University, Nanjing, 211189, P.R. China. \\ Accepted 21 August, 2013
}

\begin{abstract}
Many traditional monopoly industries are pervaded by the external e-commerce in recent years. It is therefore important to address the internet in these special industries. We establish a model under the monopoly with the external e-commerce and the corresponding properties are achieved. This paper shows that it is advantageous for the e-commerce if there are more consumers with internet prone. The effects of the change of the market size are also discussed and we find that this change has more effects on the offline sales. In essence, we characterize the e-commerce with the industrial organization theory in this work.
\end{abstract}

Key words: Monopoly, Industrial organization, e-commerce, game theory.

\section{INTRODUCTION}

Electronic commerce, commonly known as the ecommerce, consists of the buying and selling of products or services over electronic systems, especially the World Wide Web. E-commerce has been extensively researched in business and management fields in the recent years and the effects of online sale have been fully addressed.

There are plenty of papers on the e-commerce. In the recent review paper (Doukidis et al., 2008; Fink et al., 2008; Hernandez-Ortega et al., 2008), e-business is captured under operational research management. The e-commerce affecting the optimal point of the particular firm was recently characterized (Kotabe et al., 2008). The competitive equilibrium in e-commerce was investigated in the paper (Dube et al., 2007). A model of consumer-toconsumer (C2C) e-commerce trust was developed and tested (Jones and Leonard, 2008). Out-of-date information in e-commerce application systems was explored ( $\mathrm{Li}$ et al., 2007). Martin and Camarero (2009) addressed the effects of risk on the consumers. Edelman (2009) reviewed online markets based on the priced products and un-priced ones. Wirtz and Lwi (Wirtz and Lwi, 2009) investigated two types of markets.

It is extremely important to consider impacts of the e- commerce on the industrial organization. Firstly, all consumers have multiple channels to obtain the detail information of the corresponding goods because of the ecommerce, which issues in the complete information. Secondly, the e-commerce reduces the pre-sales costs. Finally, the e-commerce is more and more popular in the society.

Monopoly is still a popular type of industrial organization all over the world. In some towns of the developing countries all over the world, for example, almost all industries lie in the monopoly position (see the introduction part of the very interesting paper of Nie (2011) for firms' strategies under monopoly. It is therefore important to further address these special industries. Many industries, such as railway, post, petroleum and so on, are still in the monopoly in some countries (Nie, 2010, 2009, 2010b).

With the advent of the e-commerce, many traditional monopoly industries therefore face new competitions. It is important for these industries to smoothly deal with this type of competition. There are many ways for these industries when they face the competition of the external e-commerce. Some traditional industries also introduce e-commerce to defend the invasion of the external ecommerce. Some industries strengthen the pre-sales to 
attract consumers as many as possible.

We focus on monopoly industries when the external ecommerce is introduced. The prices of online products and the offline products to sell are respectively characterized. The previous works on the e-commerce mainly lie in the management field. We aim to develop the properties of the e-commerce in industrial organization theory in this paper, which is the main contribution of this work.

This paper is organized as follows: The model is given in Section 2. The properties of the model in Section 2 are analyzed in Section 3. Some remarks are given in the final section.

\section{The model}

We give the model formally, here. $N$ denotes the number of consumers or the market size, which is a constant in this paper. We further point out that we just consider the advent of the e-commerce at some time. It is therefore rational to assume that $N$ is always fixed. The integer $W$ denotes the number of the consumers buying online and $N-W$ denotes the number of potential offline consumers. The integer $M$ denotes the number of the consumers with internet prone and $N-M$ is the number of the consumers with less internet prone.

Similar to the analysis in Pfeil et al. (2008), we assume the variable costs of the e-commerce are given by the following formulation

$c^{\text {on }}(W)= \begin{cases}c_{1} & 0 \leq W \leq M, \\ c_{2} & M<W \leq N .\end{cases}$

Furthermore, we assume that there exists no pre-sales costs in online selling and the post-sales service cost satisfying (1) with $c_{1}<c_{2}$. The prices of the online and the offline are assumed to be $p^{o n}$ and $p^{\text {off }}$, respectively. In general, $p^{o n}<p^{o f f}$.The probability to success with offline is assumed to be $p\left(p^{o n}, p^{o f f}\right)$. Assume the fixed costs for the online and the offline are $F^{\text {on }}$ and $F^{\text {off }}$, respectively. It is pointed out that the parameter $W$ depends on $p^{o n}$ and $M$ satisfying $\frac{\partial W}{\partial p^{o n}}<0$ and $\frac{\partial W}{\partial M}>0$. We further assume that $\frac{\partial^{2} W}{\partial p^{o n} \partial W}=0 . \frac{\partial W}{\partial p^{o n}}<0$ means that the higher online prices issue in the decrease of the online consumers. This is rational because more consumers with internet prone result in more consumers buying online. There exists such type of $W$ meeting the above hypothesis. For example, the linear demand with the ecommerce satisfies all these assumptions. The profits of the ecommerce are therefore given by the following formulation.

$$
\pi^{o n}=W\left(p^{o n}, M\right) p^{o n}-W\left(p^{o n}, M\right) c^{o n}\left(W\left(p^{o n}, M\right)\right)-F^{o n}
$$

Let $p_{1}$ and $p_{2}$ be the probability of success to buy the product for the internet prone consumers and the less internet prone consumers. The parameter $p_{1}=p_{1}\left(p^{o n}, p^{\text {off }}\right)$ depends on $p^{\text {on }}$ and $p^{\text {off }}$, and the parameter $p_{2}=p_{2}\left(p^{\text {off }}\right)$ depends on $p^{\text {off }}$.Denote $p\left(p^{o n}, p^{o f f}\right)=\left\{\begin{array}{cc}\frac{1}{N-W}\left[(M-W) p_{1}\left(p^{o n}, p^{o f f}\right)+(N-M) p_{2}\left(p^{o f f}\right)\right] & 0 \leq W \leq M \\ p_{2}\left(p^{o f f}\right) & M<W<N \\ 0 & W=N\end{array}\right.$.

Let $c^{\text {off }}(W)=c_{0}$ denote the variable costs with offline selling. In general, $p_{2}\left(p^{\text {off }}\right) p^{\text {off }}>c_{0}$, which manifests that there are enough consumers with less internet prone. The profits of the offline firms are therefore given as follows.

$\pi^{\text {off }}=p\left(p^{o n}, p^{o f f}\right)(N-W) p^{o f f}-(N-W) c^{o f f}(W)-F^{o f f}$

(2) and (3) constitute a Betrand game. Two firms (one with internet and the other with offline) in some industry, simultaneously giving the prices, compete for $N$ potential consumers to achieve profits as much as possible. In the formulations (2) and (3), the parameter $W$ mainly depends on the prices of the online along with the number of the consumers with internet prone, and it is rational to denote $W\left(p^{\text {on }}, M\right)$.

The above model resorts to the technology of variable costs of the e-commerce in Pfeil et al. (2008). Moreover, consumers in two groups are also lent from Pfeil et al. (2008).Comparing with Pfeil et al. (2008), this paper highlights monopoly industrial, while Pfeil et al. (2008) focused on firms' strategies.

\section{MAIN RESULTS}

We here consider the model (2) -(3). To analyze the above model, the following assumptions to guarantee the existence and the unique of the solution to (2)-(3) are given.

\section{Assumption 1}

(A) $\quad \pi^{\text {on }}=W\left(p^{o n}, M\right) p^{o n}-W\left(p^{o n}, M\right) c^{o n}\left(W\left(p^{o n}, M\right)\right)-F^{o n}$ is concave in $p^{o n}$.

(B) $\pi^{\text {of } f}=p\left(p^{o n}, p^{o f f}\right)(N-W) p^{o f f}-(N-W) c^{o f f}(W)-F^{o f f}$ is concave in $p^{o f f}$.

(C) $p_{2}\left(p^{o f f}\right) p^{o f f}-c_{0}>0$ and $p^{o n}-c_{2}>0$.

(D) $\frac{\partial^{2} W}{\partial p^{o n} \partial M}=0$ and $\frac{\partial^{2} p_{1}\left(p^{o n}, p^{o f f}\right)}{\partial p^{o f f} \partial p^{o n}}=0$.

(E) $p_{1}\left(p^{o n}, p^{o f f}\right)<p_{2}\left(p^{o f f}\right)$ and $\frac{\partial p_{1}\left(p^{o n}, p^{o f f}\right)}{\partial p^{o f f}}<\frac{d p_{2}\left(p^{o f f}\right)}{d p^{o f f}}$.

In the above assumption, (A) and (B) are the sufficient conditions for the existence of the model (2)-(3). (C) is also rational for the existence of the e-commerce and the offline firm. (D) is satisfied for many demand functions, such as the linear demand. $(E)$ is rational because there are two choices for the consumers with internet prone.

For the model (2)-(3) under the above assumptions, by virtue of the envelop theorem for the profit functions for these two players, the following results hold.

Proposition 1. Assume the profits of the equilibrium to 
be $\pi^{o n, *}$ and $\pi^{o f f, *}$, respectively. We then have the following conclusion

$\frac{\partial \pi^{o n, *}}{\partial N}=0, \frac{\partial \pi^{o n, *}}{\partial W}>0, \frac{\partial \pi^{o n, *}}{\partial M}>0, \frac{\partial \pi^{o f f, *}}{\partial N}>0, \frac{\partial \pi^{o f f, *}}{\partial W}<0, \frac{\partial \pi^{o f f, *}}{\partial M}<0$.

Proof: $\frac{\partial \pi^{o n, *}}{\partial N}=0$ is directly derived from (2). We here consider $\frac{\partial \pi^{o n, *}}{\partial W}>0$. For (2), by virtue of the envelop theorem, we immediately obtain the following conclusion: $0 \leq W \leq M$ indicates

$\frac{\partial \pi^{o n, *}}{\partial W}=p^{o n}-c_{1}>0$.

If $M<W \leq N$, by direct calculation we can obtain that $\frac{\partial \pi^{o n, *}}{\partial W}=p^{o n}-c_{2}>0$. In summary, we have $\frac{\partial \pi^{o n, *}}{\partial W}>0$.

If $0 \leq W \leq M$, then $\frac{\partial \pi^{o n, *}}{\partial M}=\left(p^{o n}-c_{1}\right) \frac{\partial W}{\partial M}>0$ because of $\frac{\partial W}{\partial M}>0$ and $(\mathrm{C})$ of Assumption 1. If $M<W \leq N$, we then attain $\frac{\partial \pi^{o n, *}}{\partial M}=\left(p^{o n}-c_{2}\right) \frac{\partial W}{\partial M}>0$. We therefore achieve $\frac{\partial \pi^{o n, *}}{\partial M}>0$ for all $W$.

We here show $\frac{\partial \pi^{o f f}, *}{\partial N}>0$.When $0 \leq W \leq M$, we consider the profit function

$$
\begin{aligned}
& \pi^{\text {off }}=p\left(p^{o n}, p^{o f f}\right)(N-W) p^{o f f}-(N-W) c^{o f f}(W)-F^{o f f} \\
& =\left[(M-W) p_{1}\left(p^{o n}, p^{o f f}\right)+(N-M) p_{2}\left(p^{o f f}\right)\right] p^{o f f}-(N-W) c_{0}-F^{o f f}
\end{aligned}
$$

We therefore obtain $\frac{\partial \pi^{o f f}, *}{\partial N}=p_{2}\left(p^{o f f}\right) p^{o f f}-c_{0}>0$ according to $p_{2}\left(p^{\text {off }}\right) p^{\text {off }}>c_{0}$. When $M<W<N$, we consider the profit function of the offline and the following formulation holds with simple calculation

$$
\begin{aligned}
& \pi^{\text {off }}=p\left(p^{o n}, p^{o f f}\right)(N-W) p^{o f f}-(N-W) c^{o f f}(W)-F^{o f f} \\
& =p_{2}\left(p^{o f f}\right)(N-W) p^{o f f}-(N-W) c_{0}-F^{o f f}
\end{aligned}
$$

We therefore obtain $\frac{\partial \pi^{o f f, *}}{\partial N}=p_{2}\left(p^{o f f}\right) p^{o f f}-c_{0}>0$. We thus obtain $\frac{\partial \pi^{o f f, *}}{\partial N}>0$.

We here show $\frac{\partial \pi^{\text {off, * }}}{\partial W}<0$. When $0 \leq W \leq M$, we consider the profit function

$$
\begin{aligned}
& \pi^{\text {off }}=p\left(p^{o n}, p^{o f f}\right)(N-W) p^{o f f}-(N-W) c^{o f f}(W)-F^{o f f} \\
& =\left[(M-W) p_{1}\left(p^{o n}, p^{o f f}\right)+(N-M) p_{2}\left(p^{o f f}\right)\right] p^{o f f}-(N-W) c_{0}-F^{o f f} .
\end{aligned}
$$

We therefore have $\frac{\partial \pi^{o f f, *}}{\partial W}=-\left[p_{2}\left(p^{o f f}\right) p^{o f f}-c_{0}\right]=-\frac{\partial \pi^{o f f, *}}{\partial N}<0$. When $M<W<N$, we consider the profit function of the offline. Similarly, we also have $\frac{\partial \pi^{o f f, *}}{\partial W}=-\frac{\partial \pi^{o f f, *}}{\partial N}<0$. Thus, $\frac{\partial \pi^{o f f, *}}{\partial W}<0$ in all cases.

We here show $\frac{\partial \pi^{o f f, *}}{\partial M}<0$. When $0 \leq W \leq M$, we have $\frac{\partial \pi^{o f f, *}}{\partial M}=-\left[p_{2}\left(p^{o f f}\right) p^{o f f}-c_{0}\right]=-\frac{\partial \pi^{o f f, *}}{\partial N}<0$.

When $M<W<N$, we also have $\frac{\partial \pi^{o f f, *}}{\partial M}=-\left[p_{2}\left(p^{o f f}\right) p^{o f f}-c_{0}\right]=-\frac{\partial \pi^{o f f, *}}{\partial N}<0$.

In summary, the results in this proposition are all obtained and the proof is therefore complete.

Remarks: $\frac{\partial \pi^{o n, *}}{\partial W}>0$ manifests that more consumers buying online cause higher profits with the e-commerce. $\frac{\partial \pi^{o n, *}}{\partial M}>0$ means that the profits of the e-commerce increase with more consumers with internet prone. On the contrary, more consumers with internet prone results in lower profits with the offline firm. According to $\frac{\partial \pi^{o n, *}}{\partial M}>0$ and $\frac{\partial \pi^{o f f, *}}{\partial M}<0$, we find that it is favorable for the e-commerce in this competition between the ecommerce and the offline firm because the number of the consumers with internet prone dramatically increases. $\frac{\partial \pi^{o f f, *}}{\partial N}>0$ manifests that more potential consumers issue in higher offline profits if other conditions are fixed. The results in (4) therefore seem extremely rational.

We here give the equilibrium states to the models (2)-(3). We discuss it in three cases, respectively, according to the value of $W$.

Case $10 \leq W \leq M$. We consider the first-order optimal conditions to (2) and (3). For (2), the first-order optimal conditions are given by the following formulation

$$
\frac{\partial \pi^{o n}}{\partial p^{o n}}=f_{1}=W\left(p^{o n}, M\right)+\frac{\partial W\left(p^{o n}, M\right)}{\partial p^{o n}}\left(p^{o n}-c_{1}\right)=0 .
$$

For (3), the first-order optimal conditions are

$$
\begin{aligned}
\frac{\partial \pi^{o f f}}{\partial p^{o f f}} & =f_{2}=(M-W) p_{1}\left(p^{o n}, p^{o f f}\right)+(M-W) \frac{\partial p_{1}\left(p^{o n}, p^{o f f}\right)}{\partial p^{o f f}} p^{o f f} \\
& +(N-M) p_{2}\left(p^{o f f}\right)+(N-M) \frac{d p_{2}\left(p^{o f f}\right)}{d p^{o f f}} p^{o f f}=0
\end{aligned}
$$

The equilibrium state is determined by the system of the equations (5)-(6) under $0 \leq W \leq M$. 
Case $2 M<W<N$. For (2), the first-order optimal conditions are given by the following formulation

$$
\frac{\partial \pi^{o n}}{\partial p^{o n}}=f_{3}=W\left(p^{o n}, M\right)+\frac{\partial W\left(p^{o n}, M\right)}{\partial p^{o n}}\left(p^{o n}-c_{2}\right)=0 .
$$

For (3), the first-order optimal conditions are

$$
\frac{\partial \pi^{o f f}}{\partial p^{o f f}}=(N-W) p_{2}\left(p^{o f f}\right)+(N-W) \frac{d p_{2}\left(p^{o f f}\right)}{d p^{o f f}} p^{o f f}=0 .
$$

Or

$$
f_{4}=p_{2}\left(p^{o f f}\right)+\frac{d p_{2}\left(p^{o f f}\right)}{d p^{o f f}} p^{o f f}=0
$$

When $M<W<N$, the equilibrium state is therefore determined by the system of the equations (7)-(9).

Case $3 W=N$. For (2), the optimal conditions are (7). For (3), the optimal conditions are given by the following equations

$$
\frac{\partial \pi^{o f f}}{\partial p^{o f f}} \equiv 0
$$

In the third case, the optimal conditions are given by the system of the equations (7) and (10).

Assume the equilibrium prices for the model (2)-(3) to be $\left(p^{o n, *}, p^{o f f, *}\right)$. We here consider the equilibrium. When the parameter varying results in the change of the equilibrium are stated, the following result holds.

Proposition 2 If $0<W<N$, we have the following conclusion

$$
\frac{\partial p^{o n, *}}{\partial M}>0, \frac{\partial p^{o n, *}}{\partial N}=0, \frac{\partial p^{o n, *}}{\partial W}>0 .
$$

\section{Proof: See Appendix A.}

Remarks: It is more convenient to employ implicit function theorem than to employ comparative static analysis, when the explicit solution is not obtained. The results in proposition describe the relation of the prices of the ecommerce and the number of all types of the consumers.

The results in proposition 2 illustrate the relations between the prices with the e-commerce and the number of the consumers. The number of the internet prone increasing may result in higher prices of the e-commerce. On the other hand, the market size seems to have no direct relation with the prices of the e-commerce.

We here consider the effects on the prices with ecommerce. When there is no e-commerce, the market structures are under monopolization under offline firm. The monopoly price is given by the solution to the following problem;
$\underset{p^{m}}{\operatorname{Max}} \quad \pi^{\mathrm{m}}=N p_{2}\left(p^{m}\right) p^{m}-N c_{0}-F^{o f f}$.

The corresponding solution to (11) is $p^{m}$. The following result holds.

Proposition 3 If $0 \leq W \leq M, p^{m}>p^{o f f, *}$ If $M<W \leq N$, $p^{m}=p^{o f f, *}$.

Proof: See Appendix A.

We here consider the prices with offline firm. The following result holds

Proposition 4 If $0<W<N$, we have the following conclusion

$$
\frac{\partial p^{o f f, *}}{\partial M} \leq 0, \frac{\partial p^{o f f, *}}{\partial N} \geq 0 \text {. }
$$

Proof: See Appendix A.

Remarks: According to the above results, the number of the internet prone increasing results in the lower prices of the offline firm. Furthermore, the offline firm directly benefits from the increase of the market size.

\section{CONCLUSION}

In this paper, the monopoly industries invaded by the external firms with internet are modeled and the theoretical results are established with industrial organization theory. Judging form the conclusions, the impact of the external e-commerce is exceedingly important because the number of the consumers with internet prone dramatically increases in the recent years. Comparing with Pfeil et al. (2008), this paper also finds that the increasing number of the internet prone results in lower prices; this paper argues the offline firm directly benefits from the increase of the market size.

E-commerce is explored by the industrial organization theory in this paper. Actually, the impacts of the e-commerce to some industry are extensive and it is important to further address it for some traditional industries.

\section{REFERENCES}

Doukidis GI, Pramatari K, Lekakos G (2008). OR and the management of electronic services, Eur. J. Operat. Res. 187(3):1296-1309.

Dube P, Liu Z, Wynter L, Xia C (2007). Competitive equilibrium in ecommerce: Pricing and outsourcing, Comput. Operat. Res. 34(12):3541-3559.

Edelman B (2009). Priced and unpriced online markets, J. Econ. Perspect. 23(3):21-36.

Fink L, Zeevi A, Te'eni D (2008). The effectiveness of online customer relations tools - Comparing the perspectives of organizations and customers, Internet Res. 18(3):211-228.

Hernandez-Ortega B, Jimenez-Martinez J, Martin-DeHoyos MJ (2008). Differences between potential new and experienced e-customers Analysis of e-purchasing behaviour, Internet Res. 18(3):248-265.

Jones K, Leonard LNK (2008). Trust in consumer-to-consumer electronic commerce, Inform. Manage. 45(2): 88-95. 
Kotabe M, Mol MJ, Murray JY (2008).Outsourcing, performance, and the role of e-commerce: A dynamic perspective, Ind. Market. Manage. 37(1):37-45.

Li P, Tu MH, Ye IL, Xia ZH (2007). Preference update for e-commerce applications: Model, language, and processing, Electronic Commerce Res. 7(1):17-44.

Martin SS, Camarero C (2009). How perceived risk affects online buying, Online Inform. Rev. 33(4):629-654.

Nie PY (2011). Maintenance commitments for monopolized goods, Prague Economic Papers 20, to appear.
Nie PY (2010). Multiple-level principal-agent model under adverse selection. Afr. J. Bus. Manage. 4(18):4042-4048.

Nie PY (2010b). Spatial technology spillover, Econ. Computat. Econ. Cybernet. Stud. Res. 44(4):213-223.

Nie PY (2009). Commitment for storable goods under vertical integration. Econ. Modell. 26(2):414-417.

Pfeil CM, PosseltT, Maschke N (2008). Incentives for sales agents after the advent of the internet. Market. Lett. 19(1):51-63.

Wirtz J, Lwin MO (2009). Regulatory focus theory, trust, and privacy concern, J. Service Res. 12(2):190-207. 


\section{Appendix A}

\section{Proof of Proposition 2}

Firstly, Case 1 with $0 \leq W \leq M$ is considered. If the optimal state is determined by (5) and (6), we here consider the properties of this state. We first give the Jacobian matrix of the system of the equations (5) and (6)

$J=\left[\begin{array}{ll}\frac{\partial f_{1}}{\partial p^{o n}} & \frac{\partial f_{1}}{\partial p^{o f f}} \\ \frac{\partial f_{2}}{\partial p^{o n}} & \frac{\partial f_{2}}{\partial p^{o f f}}\end{array}\right]$

Because $\pi^{\text {on }}$ is concave in $p^{o n}$ and $\pi^{\text {of }}$ is concave in $p^{o f f}$, we have $\frac{\partial f_{1}}{\partial p^{o n}}<0$ and $\frac{\partial f_{2}}{\partial p^{o f f}}<0$. According to (5), we have $\frac{\partial f_{1}}{\partial p^{o f f}}=0$. It is obvious that $|J|>0$ according to $\frac{\partial f_{1}}{\partial p^{o n}}<0, \frac{\partial f_{2}}{\partial p^{o f f}}<0$ and $\frac{\partial f_{1}}{\partial p^{o f f}}=0$.

According to the implicit function theorem, there exist the solutions $p^{o n}(W, M, N)$ and $p^{o f f}(W, M, N)$, which are all differential to the parameter $W, M$ and $N$ to the system of the equations (5)-(6). According to (5), $\frac{\partial f_{1}}{\partial p^{o f f}}=0$, $\frac{\partial f_{2}}{\partial p^{o f f}}<0,|J|>0$ and the assumption of $\frac{\partial^{2} W}{\partial p^{o n} \partial M}=0$, we further obtain $\frac{\partial f_{1}}{\partial M}=\frac{\partial W}{\partial M}$ and the following formulations hold.

$$
\begin{gathered}
\frac{\partial p^{o n, *}}{\partial M}=-\frac{\left|\begin{array}{ll}
\frac{\partial f_{1}}{\partial M} & \frac{\partial f_{1}}{\partial p^{o f f}} \\
\frac{\partial f_{2}}{\partial M} & \frac{\partial f_{2}}{\partial p^{o f f}}
\end{array}\right|}{|J|}=-\frac{\frac{\partial f_{1}}{\partial M} \frac{\partial f_{2}}{\partial p^{o f f}}}{|J|}=-\frac{\frac{\partial W}{\partial M} \frac{\partial f_{2}}{\partial p^{o f f}}}{|J|}>0 . \\
\frac{\partial p^{o n, *}}{\partial N}=-\frac{\left|\begin{array}{ll}
\frac{\partial f_{1}}{\partial N} & \frac{\partial f_{1}}{\partial p^{o f f}} \\
\frac{\partial f_{2}}{\partial N} & \frac{\partial f_{2}}{\partial p^{o f f}}
\end{array}\right|}{|J|}=-\frac{\frac{\partial f_{1}}{\partial N} \frac{\partial f_{2}}{\partial p^{o f f}}}{|J|}=-\frac{\frac{\partial W}{\partial N} \frac{\partial f_{2}}{\partial p^{o f f}}}{|J|}=0 . \\
\frac{\partial p^{o n, *}}{\partial W}=-\frac{\left|\begin{array}{ll}
\frac{\partial f_{1}}{\partial W} & \frac{\partial f_{1}}{\partial p^{o f f}} \\
\frac{\partial f_{2}}{\partial W} & \frac{\partial f_{2}}{\partial p^{o f f}}
\end{array}\right|}{|J|}=-\frac{\frac{\partial f_{1}}{\partial W} \frac{\partial f_{2}}{\partial p^{o f f}}}{|J|}=-\frac{\frac{\partial f_{2}}{\partial p^{o f f}}}{|J|}>0 .
\end{gathered}
$$

The final equation lies in $\frac{\partial W}{\partial N}=0$, which results from (5). Therefore, the conclusions hold for the case $0 \leq W \leq M$. Caes $2 M<W<N$. If the optimal state is determined by (7)-(9), we here consider this situation. We also have $\frac{\partial f_{3}}{\partial p^{o n}}<0$ and $\frac{\partial f_{4}}{\partial p^{o f f}}<0$. Let $\bar{J}=\left[\begin{array}{ll}\frac{\partial f_{3}}{\partial p^{o n}} & \frac{\partial f_{3}}{\partial p^{o f f}} \\ \frac{\partial f_{4}}{\partial p^{o n}} & \frac{\partial f_{4}}{\partial p^{o f f}}\end{array}\right]$. According to (7), we have $\frac{\partial f_{3}}{\partial p^{o f f}}=0$ and $|\bar{J}|>0$. By the similar analysis to case (1) , we further have the following formulations.

$$
\begin{aligned}
& \frac{\partial p^{o n, *}}{\partial M}=-\frac{\left|\begin{array}{ll}
\frac{\partial f_{3}}{\partial M} & \frac{\partial f_{3}}{\partial p^{o f f}} \\
\frac{\partial f_{4}}{\partial M} & \frac{\partial f_{4}}{\partial p^{o f f}}
\end{array}\right|}{|\bar{J}|}=-\frac{\frac{\partial f_{3}}{\partial M} \frac{\partial f_{4}}{\partial p^{o f f}}}{|\bar{J}|}=-\frac{\frac{\partial W}{\partial M} \frac{\partial f_{4}}{\partial p^{o f f}}}{|\bar{J}|}>0 . \\
& \frac{\partial p^{o n, *}}{\partial N}=-\frac{\left|\begin{array}{ll}
\frac{\partial f_{3}}{\partial N} & \frac{\partial f_{3}}{\partial p^{o f f}} \\
\frac{\partial f_{4}}{\partial N} & \frac{\partial f_{4}}{\partial p^{o f f}}
\end{array}\right|}{|\bar{J}|}=-\frac{\frac{\partial f_{3}}{\partial N} \frac{\partial f_{4}}{\partial p^{o f f}}}{|\bar{J}|}=-\frac{\frac{\partial W}{\partial N} \frac{\partial f_{4}}{\partial p^{o f f}}}{|\bar{J}|}=0 . \\
& \frac{\partial p^{o n, *}}{\partial W}=-\frac{\left|\begin{array}{ll}
\frac{\partial f_{3}}{\partial W} & \frac{\partial f_{3}}{\partial p^{o f f}} \\
\frac{\partial f_{4}}{\partial W} & \frac{\partial f_{4}}{\partial p^{o f f}}
\end{array}\right|}{|\bar{J}|}=-\frac{\frac{\partial f_{3}}{\partial W} \frac{\partial f_{4}}{\partial p^{o f f}}}{|\bar{J}|}=-\frac{\frac{\partial f_{4}}{\partial p^{o f f}}}{|\bar{J}|}>0
\end{aligned}
$$

The results hold when $M<W<N$.

In summary, the conclusions hold for $0<W<N$ and the proof is therefore complete.

\section{Proof of Proposition 3}

By virtue of the first-order optimal conditions to (11), the monopoly prices $p^{m}$ are given by the following equation

$$
p_{2}\left(p^{m}\right)+\frac{d p_{2}\left(p^{m}\right)}{d p^{m}} p^{m}=0 .
$$

Furthermore, we have $p_{2}(p)+\frac{d p_{2}(p)}{d p} p\left\{\begin{array}{lll}>0 & \text { if } & p<p^{m} \\ >0 & \text { if } & p>p^{m}\end{array}\right.$ because $\pi^{m}$ is concave and $p^{m}$ is the optimal solution to (11).

According to $0 \leq W \leq M$, (E) of the Assumption 1 and the equation (6),we immediately have $p_{2}\left(p^{o f f}\right)+\frac{d p_{2}\left(p^{o f f}\right)}{d p^{o f f}} p^{o f f}>0$ and 
$p_{1}\left(p^{o n}, p^{o f f}\right)+\frac{\partial p_{1}\left(p^{o n}, p^{o f f}\right)}{\partial p^{o n}} p^{o n}<0$. We therefore have $p^{m}>p^{o f f, *}$ if $0 \leq W \leq M$.

If $M<W \leq N$, compared (A2) and (9), we obtain that $p^{m}=p^{\text {off }, *}$ by virtue of the concave of $\pi^{m}$.

The result in this proposition holds and the proof is therefore complete.

\section{Proof of Proposition 4}

We here consider case 1 with $0 \leq W \leq M$. Similar to the proof in Proposition 2, we have $|J|>0$ and there exist the solutions $p^{o n}(W, M, N)$ and $p^{o f f}(W, M, N)$, which are all differential to the parameter $W, M$ and $N$ to the system of the equations (5)-(6).

$\frac{\partial p^{o f f}, *}{\partial M}=-\frac{\left|\begin{array}{ll}\frac{\partial f_{1}}{\partial p^{o n}} & \frac{\partial f_{1}}{\partial M} \\ \frac{\partial f_{2}}{\partial p^{o n}} & \frac{\partial f_{2}}{\partial M}\end{array}\right|}{|J|}<0$.

The inequality holds because $\frac{\partial f_{1}}{\partial p^{o n}}<0, \frac{\partial f_{1}}{\partial M}>0, \frac{\partial f_{2}}{\partial p^{o n}}<0$ and $\frac{\partial f_{2}}{\partial M}<0$, where $\frac{\partial f_{2}}{\partial M}=\left[p_{1}\left(p^{o n}, p^{o f f}\right)+\frac{\partial p_{1}\left(p^{o n}, p^{o f f}\right)}{\partial p^{o n}} p^{o n}\right]$ $-\left[p_{2}\left(p^{o f f}\right)+\frac{d p_{2}\left(p^{o f f}\right)}{d p^{o f f}} p^{o f f}\right]<0$

from

$p_{2}\left(p^{o f f}\right)+\frac{d p_{2}\left(p^{o f f}\right)}{d p^{o f f}} p^{o f f}>0$

and

$p_{1}\left(p^{o n}, p^{o f f}\right)+\frac{\partial p_{1}\left(p^{o n}, p^{o f f}\right)}{\partial p^{o n}} p^{o n}<0$ in Proposition 3. $\frac{\partial f_{2}}{\partial p^{o n}}=$ $(M-W)\left[\frac{\partial p_{1}\left(p^{o n}, p^{o f f}\right)}{\partial p^{o n}} \quad+\frac{\partial^{2} p_{1}\left(p^{o n}, p^{o f f}\right)}{\partial p^{o n} \partial p^{o f f}}\right]=$ $(M-W) \frac{\partial p_{1}\left(p^{o n}, p^{o f f}\right)}{\partial p^{o n}}<0 . \quad \frac{\partial f_{1}}{\partial p^{o n}}<0$ and $\frac{\partial f_{1}}{\partial M}>0$ are all obtained in Proposition 2.

$\frac{\partial p^{o f f, *}}{\partial N}=-\frac{\left|\begin{array}{ll}\frac{\partial f_{1}}{\partial p^{o n}} & \frac{\partial f_{1}}{\partial N} \\ \frac{\partial f_{2}}{\partial p^{o n}} & \frac{\partial f_{2}}{\partial N}\end{array}\right|}{|J|}>0$ because $\quad \frac{\partial f_{1}}{\partial p^{o n}}<0, \quad \frac{\partial f_{2}}{\partial p^{o n}}<0, \quad \frac{\partial f_{1}}{\partial N}=0 \quad$ and $\quad \frac{\partial f_{2}}{\partial N}=$ $p_{2}\left(p^{\text {off }}\right)+\frac{d p_{2}\left(p^{\text {off }}\right)}{d p^{\text {off }}} p^{\text {off }}>0$.

The results hold when $0 \leq W \leq M$. Here we show that the results also hold when $M<W<N$.Similarly, we have $\frac{\partial f_{3}}{\partial p^{o n}}<0$ and $\frac{\partial f_{4}}{\partial p^{o f f}}<0$. Let $\bar{J}=\left[\begin{array}{ll}\frac{\partial f_{3}}{\partial p^{o n}} & \frac{\partial f_{3}}{\partial p^{o f f}} \\ \frac{\partial f_{4}}{\partial p^{o n}} & \frac{\partial f_{4}}{\partial p^{o f f}}\end{array}\right]$. According to (7), we have $\frac{\partial f_{3}}{\partial p^{o f f}}=0$ and $|\bar{J}|>0$. By the similar analysis to case (1) , we further have the following formulations.

$\frac{\partial p^{o f f}, *}{\partial M}=-\frac{\left|\begin{array}{ll}\frac{\partial f_{3}}{\partial p^{o n}} & \frac{\partial f_{3}}{\partial M} \\ \frac{\partial f_{4}}{\partial p^{o n}} & \frac{\partial f_{4}}{\partial M}\end{array}\right|}{|\bar{J}|}=-\frac{\frac{\partial f_{4}}{\partial M} \frac{\partial f_{3}}{\partial p^{o n}}}{|\bar{J}|}=0$

because $\frac{\partial f_{4}}{\partial M}=0$ and $\frac{\partial f_{4}}{\partial p^{o n}}=0$.Similarly,

$\frac{\partial p^{o f f, *}}{\partial N}=-\frac{\left|\begin{array}{ll}\frac{\partial f_{3}}{\partial p^{o n}} & \frac{\partial f_{3}}{\partial N} \\ \frac{\partial f_{4}}{\partial n} & \frac{\partial f_{4}}{\partial N}\end{array}\right|}{|\bar{J}|}=-\frac{\frac{\partial f_{4}}{\partial N} \frac{\partial f_{3}}{\partial p^{o n}}}{|\bar{J}|}=0$

because $\frac{\partial f_{4}}{\partial N}=0$ and $\frac{\partial f_{4}}{\partial p^{o n}}=0$.

In summary, the conclusions hold for $0<W<N$ and the proof is therefore complete. 